\title{
Factors Associated with Caregiver Burden in Patients with Alzheimer's Disease
}

\author{
Hyo Shin Kang ${ }^{1 *}$, Woojae Myung ${ }^{2 *}$, Duk L. Na ${ }^{3}$, Seong Yoon Kim, Jae-Hong Lee ${ }^{5}$, \\ Seol-Heui $\mathrm{Han}^{6}$, Seong Hye $\mathrm{Choi}^{7}$, SangYun $\mathrm{Kim}^{8}$, Seonwoo $\mathrm{Kim}^{9}$, and Doh Kwan $\mathrm{Kim}^{1,2 匹}$ \\ ${ }^{1}$ Center for Clinical Research, Samsung Biomedical Research Institute, Seoul, Republic of Korea \\ 2Department of Psychiatry, Samsung Medical Center, Sungkyunkwan University School of Medicine, Seoul, Republic of Korea \\ ${ }^{3}$ Department of Neurology, Samsung Medical Center, Sungkyunkwan University School of Medicine, Seoul, Republic of Korea \\ ${ }^{4}$ Department of Psychiatry, Asan Medical Center, University of Ulsan College of Medicine, Seoul, Republic of Korea \\ ${ }^{5}$ Department of Neurology, Asan Medical Center, University of Ulsan College of Medicine, Seoul, Republic of Korea \\ ${ }^{6}$ Department of Neurology, Konkuk University School of Medicine, Seoul, Republic of Korea \\ ${ }^{7}$ Department of Neurology, Inha University School of Medicine, Incheon, Republic of Korea \\ ${ }^{8}$ Department of Neurology, Seoul National University College of Medicine, Seoul, Republic of Korea \\ ${ }^{9}$ Biostatistics Unit, Samsung Biomedical Research Institute, Seoul, Republic of Korea
}

Objective Caregivers for patients with Alzheimer's disease $(\mathrm{AD})$ suffer from psychological and financial burdens. However, the results of the relationship between burden and cognitive function, performance of activities of daily living, and depressive symptoms have remained inconsistent. Therefore, the aim of this study was to examine which factors are more significant predictors of heightened burden, cognitive impairment or functional decline, besides neuropsychiatric symptoms.

Methods A cross-sectional study was conducted in a sample comprised of 1,164 pairs of patients with AD and caregivers from the Clinical Research of Dementia of South Korea study cohorts. The cognitive function of each sub-domain, functional impairments, depressive symptoms, and caregiver burden were assessed using the dementia version of Seoul Neuropsychological Screening Battery (SNSB-D), Barthel Index for Daily Living Activities (ADL), Seoul-Instrumental Activities of Daily Living (S-IADL), the Clinical Dementia Rating Sum of Box (CDR-SB), the Global Deterioration Scale (GDS), the Korean version of the Neuropsychiatric Inventory (K-NPI), and the 15 -item Geriatric Depression Scale.

Results We found that higher severity (higher CDR-SB and GDS scores) and more functional impairment (lower ADL and higher SIADL scores) were significantly associated with higher caregiver burden. In addition, depressive symptoms of patients (higher Geriatric Depression Scale scores) were associated with higher caregiver burden.

Conclusion Therefore, interventions to help maintain activities of daily living in patients with AD may alleviate caregiver burden and improve caregiver well-being.

Psychiatry Investig 2014;11(2):152-159

Key Words Caregiver, Alzheimer's disease, Cognition, Activities of daily living.

\section{INTRODUCTION}

Alzheimer's disease $(\mathrm{AD})$ is a progressive and degenerative disease of the brain that causes multiple cognitive areas to be-

Received: April 25, 2013 Revised: July 30, 2013

Accepted: August 7, 2013 Available online: April 11, 2014

$\triangle$ Correspondence: Doh Kwan Kim, MD, PhD

Department of Psychiatry, Samsung Medical Center, Sungkyunkwan University School of Medicine, 81 Irwon-ro, Gangnam-gu, Seoul 135-710, Republic of Korea

Tel: +82-2-3410-3582, Fax: +82-2-3410-0941, E-mail: paulkim@skku.edu

*These individuals contributed equally to this article as co-first authors.

(a) This is an Open Access article distributed under the terms of the Creative Commons Attribution Non-Commercial License (http://creativecommons.org/licenses/by$\mathrm{nc} / 3.0$ ) which permits unrestricted non-commercial use, distribution, and reproduction in any medium, provided the original work is properly cited. come seriously impaired and results in a decline in functional abilities and behavioral changes. The prevalence of $\mathrm{AD}$ among persons aged $>65$ years is $2.7-11.2 \%$, and the annual costs for $\mathrm{AD}$ are $\$ 20$ to $\$ 100$ billion in the United States. ${ }^{1,2}$ As the prevalence and costs of $\mathrm{AD}$ grow rapidly, the burden to family, society, and the nation increases.

In particular, primary caregivers frequently suffer from enormous psychological and financial stress. This stress makes caregivers choose to institutionalize patients with $\mathrm{AD}$ earlier and, consequently, the burden on social and health services increases. ${ }^{3,4}$ Thus, it is important to focus on caregiver burden, because it increases social costs and the individual burden of dementia. 
Several studies have been conducted with the aim of identifying factors related to caregiver burden. Mohamed et al. ${ }^{3}$ found that severity of psychiatric symptoms, behavioral disturbances, and patient quality of life have a stronger association with caregiver-experienced burden. Kim et al. ${ }^{5}$ reported that caregiver-related factors, such as gender, history of home care, and education level are correlated with caregiver burden. Additionally, patient-related factors such as performance of activities of daily living (ADLs) have been related to burden.

Although many studies have tried to show the factors associated with caregiver burden, there is a lack of comprehensive studies that have investigated which factor is the most significant predictor of caregiver burden. Many studies have focused on caregiver or patient-related sociodemographic characteristics, ${ }^{4-6}$ but few studies have conducted comprehensive neuropsychological testing and examined cognitive function subdomains to identify the association between patient cognitive function and caregiver burden.

We designed this study to explore specific factors that are associated with caregiver burden and to integrate current findings. In this study, we examined whether cognitive dysfunction or functional impairment is more related to caregiver burden. In addition, we examined the association among caregiver burden and demographic variables of patients and caregivers, patient depressive symptoms, insight, and underlying medical conditions using multivariate analysis in a large cohort of multicenter patients.

\section{METHODS}

\section{Subjects and study design}

The Clinical Research of Dementia of South Korea (CREDOS) study is a 9-year longitudinal, nationwide, hospitalbased study that has been carried out in 56 hospitals. This study had a cross-sectional design. The sample comprised 1,164 patients diagnosed with incident $\mathrm{AD}$ and 1,164 caregivers. This study was based on baseline data derived from the CREDOS study from February 2005 to February 2011. All patients were diagnosed with $\mathrm{AD}$ or probable $\mathrm{AD}$ using the criteria of the National Institute of Neurological and Communicative Disorders and Stroke-Alzheimer's Disease and Related Disorders Association (NINCDS-ADRDA). ${ }^{7}$

Patients were excluded if they had any neurodegenerative disease other than AD (dementia with Lewy bodies, Parkinson's disease, Huntington's disease, etc.). Patients with mild cognitive impairment were also excluded. Subjects with a severe medical condition (e.g., uncontrolled diabetes mellitus, severe liver failure, severe arrhythmia, and end-stage renal disease) were excluded due to safety issues. Additionally, we excluded subjects with significant hearing or visual impairments that rendered interviews difficult. Each caregiver met the following inclusion criteria: 1) was a relative of the patient; 2) was familiar with the patient's daily activities; and 3) agreed to be interviewed.

This study protocol was approved by the Institutional Review Boards of all participating hospitals (\#2005-02-008). Signed informed consent was obtained from both caregivers and patients.

\section{Dementia assessment}

The clinical workup included complete medical histories, physical and neurological evaluations, routine laboratory tests, including complete blood counts, blood chemistry profiles, vi$\operatorname{tamin} \mathrm{B}_{12} /$ folate levels, syphilis serology, thyroid function tests, and a brain magnetic resonance imaging (MRI) evaluation. Genotyping for the APOE polymorphism was undertaken in 733 subjects. Brain MRI scans included transaxial T2, T1weighted scans, and fluid-attenuated inversion recovery slices. CREDOS rating scales were employed to assess white matter changes. ${ }^{8}$ Patients were classified into three groups (mild, moderate, and severe) based on their white matter changes around the lateral ventricles or in deep white matter.

All patients underwent a standardized neuropsychological battery (the dementia version of Seoul Neuropsychological Screening Battery, SNSB-D) (Table 1). ${ }^{9}$ The SNSB-D consists of sub-domains that assess attention (score of 0-17), language and related function (score of $0-27$ ), visuospatial function (score of 0-36), memory (score of 0-150) and frontal/executive function (score of 0-70). Higher scores indicate intact function in all sub-domains. The Barthel Index for Daily Living Activities (rating 0-20, higher scores indicate more independence) was used to evaluate basic ADLs. ${ }^{10}$ We used the Seoul-Instrumental Activities of Daily Living (S-IADL) for the instrumental ADL tasks. ${ }^{11,12}$ The S-IADL was developed to assess patient ability to perform social and instrumental ADLs. All items were scored on a 4-point scale ranging from 0 to 3 , and the possible score ranged from 0 to 24 . A lower score indicates better functioning. The Korean version of the Neuropsychiatric Inventory (K-NPI) was administrated to the caregivers of the subjects. ${ }^{13}$ The K-NPI evaluates 12 domains of common behavioral and psychological symptoms, including delusions, hallucinations, agitation/aggression, depression/dysphoria, anxiety, euphoria/elation, apathy/indifference, disinhibition, irritability/lability, aberrant motor behavior, sleep/nighttime disturbances, and appetite/eating abnormalities. The caregivers were asked about symptoms that had occurred within the preceding 4 weeks. After the questions were answered, the caregivers were asked about symptom frequency (scale of 1 to 4 , higher scores indicate more frequent) as well as severity (scale of 1 to 3 , higher 
scores indicate more severe). The composite score (rating, 0-12) for each domain was calculated by multiplying frequency by severity. A total score (range, $0-144$ ) was calculated by adding the 12 composite scores. Depressed mood was assessed using the 15-item Geriatric Depression Scale, ${ }^{14}$ and the Korean version of the Mini-Mental State Examination (K-MMSE) was used to assess cognitive impairment. ${ }^{15,16}$ Global severity of disease was assessed using the Clinical Dementia Rating Sum of Box (CDR-SB) ${ }^{17}$ and the Global Deterioration Scale (GDS).$^{18}$

\section{Caregiver assessment}

Caregiver burden was evaluated using the Neuropsychiatric Inventory Caregiver Distress Scale (NPI-D). ${ }^{19}$ The NPI-D was developed to provide a quantitative measure of distress experienced by caregivers in relation to the individual symptom domains assessed by the K-NPI. After rating each symptom item of the K-NPI, caregivers were asked to rate their distress (range, $0-5$, higher score indicates more distress). The NPI$\mathrm{D}$ has a moderate correlation $(\mathrm{r}=0.51, \mathrm{p}<0.01)$ with the Zarit Caregiver Burden Inventory (ZBI), which is a widely used assessment scale for measuring caregiver perceived burden as reported by caregivers. ${ }^{20,21}$

Table 1. Construction of the SNSB-D

\begin{tabular}{|c|c|c|}
\hline Domains & Scores & Subtests \\
\hline Attention & 17 & $\begin{array}{l}\text { Digit span forward } \\
\text { Digit span backward }\end{array}$ \\
\hline $\begin{array}{l}\text { Language and related } \\
\text { function }\end{array}$ & 27 & $\begin{array}{l}\text { Short form of K-BNT (A form) } \\
\text { Calculation }\end{array}$ \\
\hline Visuospatial function & 36 & RCFT copy \\
\hline Memory & 150 & $\begin{array}{l}\text { Orientation } \\
\text { SVLT free/delayed recalls } \\
\text { SVLT recognition } \\
\text { RCFT immediate/delayed recalls } \\
\text { RCFT recognition }\end{array}$ \\
\hline $\begin{array}{l}\text { Frontal/Executive } \\
\text { function }\end{array}$ & 70 & $\begin{array}{l}\text { Motor impersistence } \\
\text { Contrasting program } \\
\text { Go-no-go test } \\
\text { Fist-edge-palm } \\
\text { Luria loop } \\
\text { Category word generation (animal) } \\
\text { Phonemic word generation }(\neg) \\
\text { Stroop test-color reading }\end{array}$ \\
\hline GCF score & 300 & \\
\hline
\end{tabular}

SNSB-D: Seoul Neuropsychological Screening Battery-Dementia Version, K-BNT: Korean-Boston Naming Test, RCFT: Rey-Complex Figure Test, SVLT: Seoul Verbal Learning Test, GCF: global cognitive function

\section{Data analysis}

Continuous variables that were not normally distributed are presented as medians and interquartile ranges. Non-parametric methods were applied to analyze these variables. We employed the Wilcoxon rank-sums test or the Kruskal-Wallis test to compare groups of continuous variables. Spearman's rank correlation test was used to test correlations between continuous variables. Median regression was employed to test the multivariate association between NPI-D and the dependent variables. We examined potential collinearity by using Variance inflation factor (VIF) scores, and we did not detect any variables with collinearity ( $\mathrm{VIF}>5$ ). Results were considered significant at $\mathrm{p}<0.05$. $\mathrm{p}$-values were corrected using Bonferronis correction if necessary.

\section{RESULTS}

\section{Subject characteristics}

The clinical and demographic characteristics of the patients are shown in Table 2. The median age of the patients was 74 years (interquartile range, 68-79 years). We recruited 445 (38.1\%) male patients and 719 (62.8\%) female patients (male: female, 1:1.62). Almost all of the patients were educated more than 6 years (median, 7 years; interquartile range, 6-12 years).

Table 2. Clinical and demographic characteristics of the subjects

\begin{tabular}{lcc}
\hline & Patients $(\mathrm{N}=1164)$ & Caregiver $(\mathrm{N}=1164)$ \\
\hline Age (years) & $74(68-79)$ & $51(42-66)$ \\
Gender (\%, male) & $445(38.1)$ & $402(34.5)$ \\
Education (years) & $7(6-12)$ & \\
ADL & $20(20-20)$ & \\
S-IADL & $15(10-23)$ & \\
K-NPI & $7(2-18)$ & \\
K-MMSE & $20(17-23)$ & \\
CDR-SB & $4.5(3-6)$ & $4(1-12)$ \\
GDS & $4(4-5)$ & \\
NPI-D & & $388(33.3)$ \\
Relationship (N, \%) & & $240(20.6)$ \\
Spouse & & $332(28.5)$ \\
Son & & $204(17.5)$ \\
Daughter & & 58.9 \\
Others & & \\
Living with patients (\%) & & \\
\hline
\end{tabular}

Continuous variables that were not normally distributed are presented as medians and interquartile ranges. ADL: activities of daily living, S-IADL: Seoul-Instrumental Activities of Daily Living, KNPI: Korean Neuropsychiatric Inventory, K-MMSE: Korean Mini Mental State Examination, CDR-SB: Clinical Dementia Rating Sum of Boxes, GDS: Global Deterioration Scale, NPI-D: Neuropsychiatric Inventory-caregiver Distress Scale 
Table 3. Correlations among clinical and demographic characteristics of patients, caregivers, and caregiver burden (NPI-D)

\begin{tabular}{|c|c|c|c|}
\hline Variables & NPI-D & Statistics & $\mathrm{p}$ \\
\hline \multicolumn{4}{|l|}{ Patients } \\
\hline \multicolumn{4}{|l|}{ Demographic } \\
\hline Age (years)* & & rho $=-0.01$ & 0.82 \\
\hline Gender (male/female $)^{\dagger}$ & $4(1,12) / 4(1,12)$ & $Z=-0.28$ & 0.78 \\
\hline Education (years)* & & rho $=-0.05$ & 0.11 \\
\hline Current alcohol drinking (drinker/non-drinker) ${ }^{\dagger}$ & $4(1,12) / 4(1,12)$ & $Z=-0.67$ & 0.50 \\
\hline Smoking history (smoker/non-smoker) ${ }^{\dagger}$ & $4(1,12) / 4(0,11)$ & $Z=-1.80$ & 0.08 \\
\hline \multicolumn{4}{|l|}{ Clinical } \\
\hline CDR-SB* & & rho $=0.37$ & $<0.0001$ \\
\hline $\mathrm{ADL}^{*}$ & & rho $=-0.25$ & $<0.0001$ \\
\hline S-IADL* & & rho $=0.42$ & $<0.0001$ \\
\hline K-MMSE* & & rho $=-0.14$ & $<0.0001$ \\
\hline GDS* & & rho $=0.30$ & $<0.0001$ \\
\hline Geriatric Depression Scale* & & rho $=0.12$ & $<0.0001$ \\
\hline Illness duration* & & rho $=-0.03$ & 0.39 \\
\hline Onset type (acute/insidious) ${ }^{\dagger}$ & $4(1,10) / 4(1,12)$ & $Z=-1.09$ & 0.27 \\
\hline Insight (impaired/partial/intact) $\ddagger$ & $7(2,15) / 4(0,10) / 5(1,12)$ & $\mathrm{X}_{2}^{2}=17.64$ & $<0.001$ \\
\hline \multicolumn{4}{|l|}{ Genetic and Imaging } \\
\hline APOE genotype $(0 / 1 / 2 \text {, number of } \mathrm{E} 4 \text { allele })^{\ddagger}$ & $4(1,12) / 5(1,11) / 6(1,14)$ & $\mathrm{X}_{2}^{2}=1.19$ & 0.55 \\
\hline \multicolumn{4}{|l|}{ Brain Ischemic change } \\
\hline Deep brain white matter change (mild/moderate/severe)‡ & $4(1,12) / 5(0,12) / 4(1,10)$ & $\mathrm{X}_{2}^{2}=0.03$ & 0.98 \\
\hline Periventricular white matter change (mild/moderate/severe) $)^{\ddagger}$ & $4(1,12) / 4(1,11) / 4(1,12)$ & $\mathrm{X}_{2}^{2}=0.26$ & 0.88 \\
\hline \multicolumn{4}{|l|}{ Neurologic Exam (with/without) } \\
\hline Motor weakness ${ }^{\dagger}$ & $7(1.5,16) / 4(1,12)$ & $Z=-1.31$ & 0.19 \\
\hline Sensory loss ${ }^{\dagger}$ & $12(0,16) / 4(1,12)$ & $Z=-0.24$ & 0.81 \\
\hline Parkinsonian feature ${ }^{\dagger}$ & $4(1.5,13) / 4(1,11)$ & $Z=-1.15$ & 0.25 \\
\hline \multicolumn{4}{|l|}{ Neuropsychological test (SNSB-D score) } \\
\hline Attention* & & rho $=-0.07$ & 0.02 \\
\hline Language* & & rho $=-0.10$ & 0.001 \\
\hline Visuospatial* & & rho $=-0.07$ & 0.01 \\
\hline Memory* & & $\mathrm{rho}=-0.14$ & $<0.0001$ \\
\hline Frontal and Executive* & & $\mathrm{rho}=-0.16$ & $<0.0001$ \\
\hline \multicolumn{4}{|l|}{ Underlying Medical History (with/without) } \\
\hline $\mathrm{DM}^{\dagger}$ & $4(1,13) / 4(1,11)$ & $\mathrm{Z}=-1.16$ & 0.25 \\
\hline $\mathrm{HTN}^{\dagger}$ & $4(1,12) / 4(1,12)$ & $Z=-0.33$ & 0.74 \\
\hline Hyperlipidemia $^{\dagger}$ & $6(1,12) / 4(1,11)$ & $Z=-1.80$ & 0.07 \\
\hline Heart disease $^{\dagger}$ & $6(1,14) / 4(1,11)$ & $Z=-2.26$ & 0.02 \\
\hline Stroke $^{\dagger}$ & $6(2,14) / 4(1,11)$ & $Z=-1.63$ & 0.10 \\
\hline Head trauma ${ }^{\dagger}$ & $6.5(2,15) / 4(1,12)$ & $\mathrm{Z}=-1.28$ & 0.20 \\
\hline $\mathrm{CO}_{\text {poisoning }}{ }^{\dagger}$ & $5(2,10) / 4(1,12)$ & $Z=-0.48$ & 0.63 \\
\hline Brain surgery ${ }^{\dagger}$ & $3.5(2,7) / 4(1,12)$ & $Z=0.56$ & 0.57 \\
\hline Syphilis $^{\dagger}$ & $20(8,24.5) / 4(1,12)$ & $Z=-1.39$ & 0.16 \\
\hline Thyroid disease $^{\dagger}$ & $4(1,10) / 4(1,12)$ & $Z=0.06$ & 0.96 \\
\hline Liver disease $^{\dagger}$ & $15(2,15) / 4(1,12)$ & $Z=-0.97$ & 0.33 \\
\hline
\end{tabular}


Table 3. Continued

\begin{tabular}{|c|c|c|c|}
\hline Variables & NPI-D & Statistics & $\mathrm{p}$ \\
\hline Renal disease $^{\dagger}$ & $9(3,19) / 4(1,11)$ & $Z=-5.56$ & $<0.0001$ \\
\hline Lung disease $^{\dagger}$ & $8(3,17) / 4(1,11)$ & $Z=-2.57$ & 0.01 \\
\hline Cancer $^{\dagger}$ & $6(1,13) / 4(1,11)$ & $Z=-2.18$ & 0.03 \\
\hline Epilepsy $^{\dagger}$ & $13(9.5,22) / 4(1,12)$ & $Z=-2.23$ & 0.03 \\
\hline \multicolumn{4}{|l|}{ Caregiver } \\
\hline Age (year)* & & rho $=-0.12$ & $<0.0001$ \\
\hline Gender (male/female) ${ }^{\dagger}$ & $8(3,19) / 6(2,16)$ & $\mathrm{Z}=3.40$ & $<0.001$ \\
\hline Relationship (spouse/son/daughter/others) ${ }^{\ddagger}$ & $6(1,17) / 6(2,17) / 8(3,21) / 10(4 / 20.5)$ & $\mathrm{X}_{3}^{2}=20.97$ & $<0.001$ \\
\hline Living (with/without) ${ }^{\dagger}$ & $7(2,18) / 7(3,17)$ & $Z=-0.59$ & 0.56 \\
\hline
\end{tabular}

Continuous variables that were not normally distributed are presented as medians and interquartile ranges. *Spearman's rank correlation test, tWilcoxon rank-sum test, ¥Kruskal-Wallis test. NPI-D: Neuropsychiatric Inventory-caregiver Distress Scale, CDR-SB: Clinical Dementia Rating Sum of Boxes, ADL: activities of daily living, S-IADL: Seoul-Instrumental Activities of Daily Living, K-MMSE: Korean Mini Mental State Examination, GDS: Global Deterioration Scale, APOE genotype: apolipoprotein E genotype, SNSB-D: Seoul Neuropsychological Screening Battery-Dementia version

The median K-NPI and K-MMSE scores were 7 (interquartile range, 2-18) and 20 (interquartile range, 17-23), respectively. The median GDS score was 4 (interquartile range, 4-5), comparable with moderate cognitive decline. The median age of the caregivers was 51 years (interquartile range, 42-66 years), and they consisted of 402 males (34.5\%) and 762 females (65.5\%). The median NPI-D score was 4 (interquartile range, $1-12$ ). In total, $33 \%$ of patient caregivers were spouses, $20.6 \%$ were sons, $28.5 \%$ were daughters, and $17.5 \%$ were others.

\section{Univariate analysis for the factors associated with caregiver burden}

No notable association with caregiver burden was found for patient age, patient gender, patient education level, or current alcohol drinking/smoking in patients (Table 3).

The patient's ability to perform ADLs was highly associated with caregiver burden. The caregivers of more dependent patients had higher NPI-D scores. The scales for dementia severity (CDR-SB and GDS) were correlated with higher caregiver burden. The Geriatric Depression Scale showed a positive correlation with NPI-D. In addition, patient insight for their disease was associated with caregiver burden. The impaired insight of patients was associated with higher caregiver burden (impaired insight group vs. partial insight group: corrected $\mathrm{p}=1.45 \times 10^{-4}$; impaired insight group vs. intact insight group: corrected $\mathrm{p}=0.02$ ). No significant difference was observed for the NPI-D score between the partial insight and intact insight groups (corrected $\mathrm{p}=0.11$ ). Patient cognitive function was associated with caregiver burden. In the SNSB$\mathrm{D}$ test, memory function and frontal and executive function were more significantly associated with caregiver burden than the other sub-domain scores. Among the underlying medical histories of patients, heart disease, renal disease, lung disease, cancer, and epilepsy were associated with caregiver burden (Table 3).

Among the caregiver-related variables, age, gender and the relationship with the patient were significantly associated with NPI-D score. However, we did not find a significant association between living with the caregiver and NPI-D score in this analysis.

Neither apolipoprotein E genotype nor brain ischemic changes were significantly associated with NPI-D score. Additionally, we did not find an association between neurological deficit (motor weakness, sensory loss, or parkinsonian features) and caregiver burden (Table 3 ).

\section{Median regression analysis for factors associated with caregiver burden}

We tested a multivariable model that included cognitive variables (SNSB-D sub-domains) and functional variables (ADL and S-IADL) as representative variables of cognitive parts and functional parts of dementia severity, respectively. In this test we adjusted for demographic characteristics, the Geriatric Depression Scale score, insight of patients, and underlying medical history, which were significant in the univariate analysis.

The results of the multivariate analysis are shown in Table 4. The more dependent patients (patients with lower ADL scores or higher S-IADL scores) had higher caregiver burden. In contrast with the result of the functional part of dementia severity, cognitive impairment in patients was not significantly associated with caregiver burden. None of the SNSB-D subdomains were significantly associated with caregiver burden.

A higher Geriatric Depression Scale score was significantly associated with higher caregiver burden in both models. Among underlying medical conditions, renal disease was sig- 
HS Kang et al.

Table 4. Median regression analysis for the factors associated with caregiver burden (NPI-D)

\begin{tabular}{|c|c|c|c|c|c|c|}
\hline & Coefficient & S.E. & $\mathrm{t}$ & $\mathrm{p}$ & 95\% CI, lower & 95\% CI, upper \\
\hline \multicolumn{7}{|l|}{ Demographic } \\
\hline Patient's age & -0.04 & 0.03 & -1.10 & 0.27 & -0.11 & 0.03 \\
\hline Patient's gender, male & 0.22 & 0.56 & 0.39 & 0.70 & -0.88 & 1.31 \\
\hline Patients education year & -0.05 & 0.05 & -1.04 & 0.30 & -0.16 & 0.05 \\
\hline \multicolumn{7}{|l|}{ Caregiver } \\
\hline Caregiver's age & -0.06 & 0.03 & -2.01 & 0.04 & -0.11 & -0.001 \\
\hline Caregiver's gender, male & -0.86 & 0.76 & -1.14 & 0.25 & -2.34 & 0.62 \\
\hline Caregiver's Relationship (spouse vs. son) & -0.35 & 1.10 & -0.32 & 0.75 & -2.50 & 1.80 \\
\hline Caregiver's Relationship (spouse vs. daughter) & -0.16 & 1.00 & -0.17 & 0.87 & -2.12 & 1.79 \\
\hline Caregiver's Relationship (spouse vs. others) & -0.25 & 1.04 & -0.24 & 0.81 & -2.29 & 1.79 \\
\hline \multicolumn{7}{|l|}{ Clinical } \\
\hline $\mathrm{ADL}$ & -0.90 & 0.12 & -7.63 & $<0.0001$ & -1.13 & -0.67 \\
\hline S-IADL & 0.29 & 0.03 & 10.74 & $<0.0001$ & 0.23 & 0.34 \\
\hline Geriatric Depression Scale & 0.17 & 0.05 & 3.34 & $<0.0001$ & 0.07 & 0.26 \\
\hline Insight & 0.38 & 0.31 & 1.22 & 0.22 & -0.23 & 0.98 \\
\hline \multicolumn{7}{|l|}{ Neuropsychological test } \\
\hline Attention & 0.08 & 0.12 & 0.68 & 0.50 & -0.15 & 0.32 \\
\hline Language & -0.03 & 0.06 & -0.50 & 0.62 & -0.15 & 0.09 \\
\hline Visuospatial & 0.03 & 0.03 & 1.20 & 0.23 & -0.02 & 0.08 \\
\hline Memory & 0.005 & 0.02 & 0.28 & 0.78 & -0.03 & 0.04 \\
\hline Frontal and Executive & 0.002 & 0.03 & 0.08 & 0.94 & -0.05 & 0.06 \\
\hline \multicolumn{7}{|l|}{ Underlying Medical History } \\
\hline Heart disease & 0.57 & 0.62 & 0.92 & 0.36 & -0.64 & 1.77 \\
\hline Renal disease & 2.47 & 0.61 & 4.03 & $<0.0001$ & 1.27 & 3.67 \\
\hline Lung disease & 0.81 & 0.99 & 0.82 & 0.41 & -1.13 & 2.75 \\
\hline Cancer & 0.44 & 0.51 & 0.86 & 0.39 & -0.57 & 1.45 \\
\hline Epilepsy & 2.99 & 2.37 & 1.26 & 0.21 & -1.67 & 7.64 \\
\hline
\end{tabular}

NPI-D: Neuropsychiatric Inventory-caregiver Distress Scale, ADL: activities of daily living, S-IADL: Seoul-Instrumental activities of daily living

nificantly associated with a higher NPI-D score. Caregiver age was significantly associated with caregiver burden. Patient insight was not significantly associated with caregiver burden.

\section{DISCUSSION}

This study examined which factors were more significant predictors of heightened burden, cognitive impairment, and functional decline. The results indicated that caregiver burden had a stronger association with functional impairment than that of cognitive functioning. In the correlation analysis, both ADLs and cognitive function were associated with caregiver burden. However, only functional ability was related to caregiver burden after controlling for other variables. In previous studies, the correlation between caregiver burden and patient ADLs and cognitive function has been controversial. For example, Mohamed et al. ${ }^{3}$ found only limited relationships among cognitive function, ADLs, and caregiver burden. Additionally, Coen et al..$^{22}$ reported that neither cognitive function nor functional impairment in patients with $\mathrm{AD}$ predicts caregiver burden, whereas Huang et al. ${ }^{23}$ found that poorer cognitive function and instrumental ADLs and ADL performance are associated with higher caregiver burden. Therefore, we controlled for other variables that could significantly impact caregiver burden to identify the association between functional ability and cognitive function. Our findings support that functional impairment of patients with $\mathrm{AD}$ was more related to caregiver burden.

Christofoletti et al. ${ }^{24}$ also suggested that caregivers of physically active patients with AD exhibit reduced burden. They noted that patients with dementia require increasing supervision and personal care depending on their functional ability, and that this results in negative effects on caregiver health. Therefore, interventions to help maintain ADLs in patients 
with $\mathrm{AD}$ can decrease caregiver burden compared to those that focus on cognitive decline. For example, Rolland et al. ${ }^{25}$ suggested that a collective exercise program leads to improved ability to perform ADLs, and that this could reduce caregiver burden.

In the present study, depressive symptoms of patients were highly associated with increased caregiver burden. This finding is similar to those of previous studies. ${ }^{3}$ Many studies have suggested that depressive symptoms are common in patients with dementia. ${ }^{26,27}$ Karttuten et al..$^{28}$ evaluated 240 patients with $\mathrm{AD}$ and showed that $32.1 \%$ of patients with very mild and $39.6 \%$ of patients with mild $\mathrm{AD}$ have depressive symptoms. Enache et al..$^{29}$ reported that $20-30 \%$ of patients with $\mathrm{AD}$ have depression.

Diagnosing depression in patients with dementia is important for adequate patient management, and depression of patients is significantly related to caregiver burden. However, diagnosing depression correctly may be complicated due to overlap of symptoms between dementia and depression..$^{29}$ Therefore, clinicians should take more notice of depressive symptoms in patients with dementia.

The results of the present study indicate that dementia patient and caregiver demographic factors did not significantly impact caregiver burden except the caregiver age was related to caregiver burden, indicating that the younger the caregiver, the greater the burden. This result is inconsistent with findings from some studies. Lim et al. ${ }^{30}$ reported that older caregivers have much more caregiver burden, because older caregivers have more stress from multiple roles than that of young caregivers. In contrast, the results of Hope et al. ${ }^{31}$ were consistent with ours. They found that younger caregivers are less committed to care because they may have jobs or greater demands from other family members. Additionally, Fitting et al. ${ }^{32}$ reported that younger caregivers are lonelier and more resentful of their role. In particular, Koreans have more sense of responsibility to care for elderly family members due to the influence of filial piety. Thus, our findings suggest that less emotional and social support in younger caregivers leads to a greater burden.

We tested the association between caregiver burden and patient underlying medical history. The results showed that renal disease was associated with high caregiver burden in patients with $\mathrm{AD}$. Renal disease is a representative chronic physical illness and caregivers of patients with chronic mental or physical illnesses experience significant distress and psychological burden. ${ }^{33}$ Our results indicate that the co-morbidity of medical illness in patients with $\mathrm{AD}$ could place additional burden on caregivers. The non-significant results of other medical history may be due to the limitation of our data, because we were unable to consider the severity, specific diag- nosis, or clinical stage of underlying medical conditions.

Several limitations should be noted in this study. It was a cross-sectional study, not a longitudinal study; thus, we could not draw conclusions regarding causality. A longitudinal study may have been more appropriate for an accurate assessment of predictors related to caregiver burden. Another limitation is that the majority of the patients were early stage $\mathrm{AD}$. Therefore, the findings may not be generalizable to more severely demented patients. In addition, we used the NPI-D to evaluate caregiver burden. This is an adjunct scale to the NPI for assessing the impact of neuropsychiatric symptoms on caregiver burden. However, this scale has a moderate correlation with the ZBI, which is the most widely used instrument for assessing caregiver distress. ${ }^{20}$ Despite these limitations, we think our findings are quite reliable, because we examined patients with $\mathrm{AD}$ in a large multicenter cohort, which was assessed comprehensively including neuropsychological testing, brain MRI, and diverse underlying medical history.

In conclusion, caregiver burden had a stronger association with functional impairment than that of cognitive functioning. Therefore, interventions to help maintain ADLs in patients with $\mathrm{AD}$ may alleviate caregiver burden and improve caregiver well-being.

\section{Acknowledgments}

D.K.K was supported by grants from the Korean Health Technology R \& D Project, Ministry of Health \& Welfare, Republic of Korea (HI10C2020), and Eisai Korea Inc. The funders had no role in study design, data collection and analysis, decision to publish, or preparation of the manuscript.

\section{Author Contributions}

Doh Kwan Kim, Hyo Shin Kang, and Woojae Myung were involved in study planning and writing of the manuscript; Doh Kwan Kim, Duk L. Na, Seong Yoon Kim, Jae-Hong Lee, Seol-Heui Han, Seong Hye Choi, and SangYun Kim were involved in CRCD study planning and conducted the clinical aspects of the study. Seonwoo Kim and Woojae Myung performed the statistical analyses.

\section{Accession Numbers}

The study is registered (NCT01198093) in ClinicalTrials.gov.

\section{REFERENCES}

1. Ernst RL, Hay JW. Economic research on Alzheimer disease: a review of the literature. Alzheimer Dis Assoc Disord 1997;11(Suppl 6):135145.

2. Trabucchi M. An economic perspective on Alzheimer's disease. J Geriatr Psychiatry Neurol 1999;12:29-38.

3. Mohamed S, Rosenheck R, Lyketsos CG, Schneider LS. Caregiver burden in Alzheimer disease: cross-sectional and longitudinal patient correlates. Am J Geriatr Psychiatry 2010;18:917-927.

4. Tew CW, Tan LF, Luo N, Ng WY, Yap P. Why family caregivers choose to institutionalize a loved one with dementia: a Singapore perspective. Dement Geriatr Cogn Disord 2010;30:509-516.

5. Kim MD, Hong SC, Lee CI, Kim SY, Kang IO, Lee SY. Caregiver burden among caregivers of Koreans with dementia. Gerontology 2009;55:106113. 
6. Kim JM, Shin IS, Jeong SJ, Gormley N, Yoon JS. Predictors of institutionalization in patients with dementia in Korea. Int J Geriatr Psychiatry 2002;17:101-106.

7. McKhann G, Drachman D, Folstein M, Katzman R, Price D, Stadlan EM. Clinical diagnosis of Alzheimer's disease: report of the NINCDSADRDA Work Group under the auspices of Department of Health and Human Services Task Force on Alzheimer's Disease. Neurology 1984;34: 939-944.

8. Moon SY, Na DL, Seo SW, Lee JY, Ku BD, Kim SY, et al. Impact of white matter changes on activities of daily living in mild to moderate dementia. Eur Neurol 2011;65:223-230.

9. Ahn HJ, Chin J, Park A, Lee BH, Suh MK, Seo SW, et al. Seoul Neuropsychological Screening Battery-dementia version (SNSB-D): a useful tool for assessing and monitoring cognitive impairments in dementia patients. J Korean Med Sci 2010;25:1071-1076.

10. Collin C, Wade DT, Davies S, Horne V. The Barthel ADL Index: a reliability study. Int Disabil Stud 1988;10:61-63.

11. Ahn IS, Kim JH, Kim S, Chung JW, Kim H, Kang HS, et al. Impairment of instrumental activities of daily living in patients with mild cognitive impairment. Psychiatry Investig 2009;6:180-184.

12. Ku HM, Kim JH, Kwon EJ, Kim SH, Lee HS, Ko HJ, et al. A study on the reliability and validity of Seoul-Instrumental Activities of Daily Living (S-IADL). J Korean Neuropsychiatr Assoc 2004;43:189-199.

13. Choi SH, Na DL, Kwon HM, Yoon SJ, Jeong JH, Ha CK. The Korean version of the neuropsychiatric inventory: a scoring tool for neuropsychiatric disturbance in dementia patients. J Korean Med Sci 2000;15: 609-615.

14. Bae JN, Cho MJ. Development of the Korean version of the Geriatric Depression Scale and its short form among elderly psychiatric patients. J Psychosom Res 2004;57:297-305.

15. Folstein MF, Folstein SE, McHugh PR. "Mini-mental state". A practical method for grading the cognitive state of patients for the clinician. J Psychiatr Res 1975;12:189-198.

16. Kang Y, Na DL, Hahn S. A validity study on the Korean Mini-Mental State Examination (K-MMSE) in dementia patients. J Korean Neurol Assoc 1997;15:300-308.

17. Morris JC. The Clinical Dementia Rating (CDR): current version and scoring rules. Neurology 1993;43:2412-2414.

18. Reisberg B, Ferris SH, de Leon MJ, Crook T. The Global Deterioration Scale for assessment of primary degenerative dementia. Am J Psychiatry 1982;139:1136-1139.

19. Kaufer DI, Cummings JL, Christine D, Bray T, Castellon S, Masterman $\mathrm{D}$, et al. Assessing the impact of neuropsychiatric symptoms in Alzheimer's disease: the Neuropsychiatric Inventory Caregiver Distress Scale. J Am Geriatr Soc 1998;46:210-215.
20. Zarit SH, Reever KE, Bach-Peterson J. Relatives of the impaired elderly: correlates of feelings of burden. Gerontologist 1980;20:649-655.

21. Huang SS, Lee MC, Liao YC, Wang WF, Lai TJ. Caregiver burden associated with behavioral and psychological symptoms of dementia (BPSD) in Taiwanese elderly. Arch Gerontol Geriatr 2012;55:55-59.

22. Coen RF, Swanwick GR, O'boyle CA, Coakley D. Behaviour disturbance and other predictors of carer burden in Alzheimer's disease. Int J Geriatr Psychiatry 1997;12:331-336.

23. Huang SS, Lee MC, Liao YC, Wang WF, Lai TJ. Caregiver burden associated with behavioral and psychological symptoms of dementia (BPSD) in Taiwanese elderly. Arch Gerontol Geriatr 2012;55:55-59.

24. Christofoletti G, Oliani MM, Bucken-Gobbi LT, Gobbi S, Beinotti F, Stella F. Physical activity attenuates neuropsychiatric disturbances and caregiver burden in patients with dementia. Clinics (Sao Paulo) 2011;66: 613-618.

25. Rolland Y, Pillard F, Klapouszczak A, Reynish E, Thomas D, Andrieu S, et al. Exercise program for nursing home residents with Alzheimer's disease: a 1-year randomized, controlled trial. J Am Geriatr Soc 2007;55: 158-165.

26. Castilla-Puentes RC, Habeych ME. Subtypes of depression among patients with Alzheimer's disease and other dementias. Alzheimers Dement 2010;6:63-69.

27. Lyketsos CG, Lopez O, Jones B, Fitzpatrick AL, Breitner J, DeKosky S. Prevalence of neuropsychiatric symptoms in dementia and mild cognitive impairment: results from the cardiovascular health study. JAMA 2002;288:1475-1483.

28. Karttunen K, Karppi P, Hiltunen A, Vanhanen M, Välimäki T, Martikainen J, et al. Neuropsychiatric symptoms and quality of life in patients with very mild and mild Alzheimer's disease. Int J Geriatr Psychiatry 2011;26:473-482.

29. Enache D, Winblad B, Aarsland D. Depression in dementia: epidemiology, mechanisms, and treatment. Curr Opin Psychiatry 2011;24:461472.

30. Lim YM, Son GR, Song JA, Beattie E. Factors affecting burden of family caregivers of community-dwelling ambulatory elders with dementia in Korea. Arch Psychiatr Nurs 2008;22:226-234.

31. Hope T, Keene J, Gedling K, Fairburn CG, Jacoby R. Predictors of institutionalization for people with dementia living at home with a carer. Int J Geriatr Psychiatry 1998;13:682-690.

32. Fitting M, Rabins P, Lucas MJ, Eastham J. Caregivers for dementia patients: a comparison of husbands and wives. Gerontologist 1986;26:248252.

33. Given CW, Given B, Stommel M, Collins C, King S, Franklin S. The caregiver reaction assessment (CRA) for caregivers to persons with chronic physical and mental impairments. Res Nurs Health 1992;15:271-283. 